\title{
The Use of Differential Scanning Calorimetry in Studies of Wax Deposition: Measuring the Solid Formation and Binary Solid-Liquid Equilibrium Phase Diagrams
}

\author{
J.A.P. Coutinho ${ }^{1 *}$ and V. Ruffier-Meray ${ }^{2}$ \\ 1 Centro de Investigação em Química, Departamento de Química, Faculdade de Ciências, Rua do Campo Alegre, 687, 4150 Porto - Portugal \\ 2 Institut français du pétrole, 1 et 4, avenue du Bois-Préau, 92852 Rueil-Malmaison Cedex - France \\ email: jcoutinho@dq.ua.pt \\ * Corresponding author. Current address: Departamento de Química, Universidade de Aveiro, 3810 Aveiro - Portugal
}

\begin{abstract}
Résumé - La DSC (Differential Scanning Calorimetry) appliquée à l'étude des dépôts de paraffines : mesure des quantités de dépôts et des diagrammes de phases (liquide-solide) de mélange binaire - L'étude de la cristallisation des paraffines dans les mélanges hydrocarbonés, qu'il s'agisse de pétroles bruts ou de produits raffinés tels que le Diesel ou le fioul, nécessite l'utilisation de techniques expérimentales permettant de déterminer le diagramme de phases. L'acquisition de ces données est primordiale, à la fois pour comprendre les mécanismes de la cristallisation et pour le développement des modèles thermodynamiques. Dans cet article, nous montrons comment l'analyse calorimétrique différentielle peut permettre d'estimer la quantité de solide déposée en fonction de la température et de mesurer les diagrammes de phases de mélanges binaires ne formant pas de solutions solides. Les résultats obtenus avec la méthode proposée s'accordent avec les données de la littérature.
\end{abstract}

Mots-clés : paraffines, dépôts, DSC, équilibres liquide-solide.

\begin{abstract}
The Use of Differential Scanning Calorimetry in Studies of Wax Deposition: Measuring the Solid Formation and Binary Solid-Liquid Equilibrium Phase Diagrams - The studies of wax formation in hydrocarbon mixtures, both crudes and refined products such as diesels and fuels, require measuring techniques able to deal with the solid phase behaviour. Experimental data on phase equilibria is important to understand the crystallisation of paraffins and for the development of thermodynamical models, that can describe it. It is here shown how the information obtained from Differential Scanning Calorimetry (DSC) measurements can be used to assess the amount of solid forming when a fluid is cooled and also to make faster measurements of phase diagrams for binary systems where no solid solutions exist. The measurements performed with the proposed methods present a good agreement with literature data.
\end{abstract}

Keywords: wax, deposit, DSC, liquid-solid equilibrium. 


\section{NOMENCLATURE}

$C_{p} \quad$ heat capacity/ $\mathrm{J} \mathrm{g}^{-1} \mathrm{~K}^{-1}$

$h$ enthalpy/J

$h^{E} \quad$ excess enthalpy/ $\mathrm{J} \mathrm{g}^{-1}$

$h_{m} \quad$ melting enthalpy $/ \mathrm{J} \mathrm{g}^{-1}$

$m$ mass/g

$q \quad$ energy/J

$T_{c} \quad$ cloud point temperature/K

$T_{\text {Eut }} \quad$ eutectic point temperature/K

$T_{m} \quad$ melting point/K

$T_{o} \quad$ starting temperature/K

W mass fraction.

\section{GREEK LETTERS}

$\alpha^{L} \quad$ fraction of melted solute

$\alpha^{S} \quad$ fraction of crystallised solute.

\section{SUBSCRIPTS AND SUPERSCRIPTS}

A component $A$

$B$ component $B$

Eut relative to the eutectic point

$i \quad$ solute $i$

liq liquid

$L \quad$ liquid

$m$ melting

sld solid

slt solute

solv solvent

S solid

total total.

\section{INTRODUCTION}

Wax formation is a problem that is present in the entire petroleum cycle, from the production and transport of crudes to the use of refined products such as diesels and fuels. To show its importance it is enough to say that additives to prevent paraffin deposition in diesels account for half the costs of the additives used. A big effort, both theoretical and experimental, has been directed in the last years in the attempt to understand the behaviour of paraffins in solution to prevent and solve the problems arising from wax deposition. One of the main obstacles that such studies still face is the lack of reliable experimental data [1] and costly and time-consuming methods for measurements concerning the solid phase.

It is here shown how a well-known technique such as DSC can be used to provide further information about the precipitation of paraffinic waxes in hydrocarbon fluids. To know how much solid will form in a fluid subjected to a given temperature is a very important information in crystallisation processes. Due to the gelling of mixtures with paraffin deposition, it is impossible to separate the phases to evaluate the amount of solids. Alternative techniques have been proposed $[2,3]$ but they are very complex requiring the establishment of the equilibrium at each temperature of interest and the measurement of the composition of the phases present. DSC can be an adequate alternative to this process allowing a much simpler and faster determination of the solid formation by analysis of the plot of enthalpy variation with temperature as described below.

Phase behaviour data for simple mixtures can also provide valuable information on wax deposition, both providing insight on the mechanisms of crystal formation as well as data for testing thermodynamic models. DSC is already widely used for phase diagram measurement but here an alternative procedure is presented. This is a bit less accurate than the conventional methods for the detection of the phase boundaries, but can provide fast and reliable phase diagrams.

\section{MEASUREMENT OF THE AMOUNT OF PRECIPITATED WAX}

When studying solid-liquid equilibria, metastable states are prone to arise. To avoid problems related with supercooled solutions it is advisable to run the experiments in heating instead of cooling. This policy was adopted all over this work and all the theoretical developments follow this procedure. The attention is thus focused on the fraction of solute(s) melted, $\alpha^{L}{ }_{s l t}$, instead of the fraction of crystallised solution, $\alpha^{S}$ solution , being the two related by Equation (1):

$$
\alpha_{\text {solution }}^{S}=\frac{m_{\text {solution }}^{S}}{m_{\text {solution }}^{\text {total }}}=\left(1-\alpha_{\text {slt }}^{L}\right) * \sum_{i} W_{\text {slti }}
$$

where $W_{\text {slti }}$ is the mass fraction of solute $i$ in the mixture. Hereafter $\alpha^{L}{ }_{\text {slt }}$ is noted $\alpha^{L}$ to simplify the notation.

The melting of a frozen mixture of $n$ solutes in a single solvent from a temperature $T_{o}$, below the solvent melting temperature, to complete fusion of the mixture, will be considered here. The approach presented below can easily be extended to other cases where a mixture of solvents no definite solvent exists. The solutes may form or not solid solutions. The aim is to measure $\alpha^{L}$ :

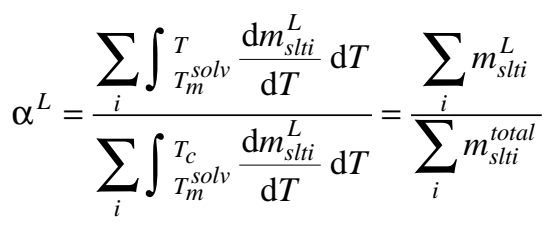


where $T_{c}$, the cloud point temperature, is the temperature of disappearance of the last crystal. Using a calorimeter, however, only enthalpic rather than mass variations can be accounted for. What can be measured by DSC is the ratio between the energy required to melt the fraction of solutes present in the liquid phase at temperature $T$, and the energy required to completely melt the solutes:

$$
\alpha^{L^{\prime}}=\frac{\sum_{i} \int_{T_{m}^{\text {solv }}}^{T} h_{m}^{\text {slti }}(T) \frac{\mathrm{d} m_{\text {slti }}^{L} \mathrm{~d} T}{\mathrm{~d} T}}{\sum_{i} \int_{T_{m}^{\text {solv }}}^{T_{c_{m}}} h_{m}^{\text {slti }}(T) \frac{\mathrm{d} m_{\text {slti }}^{L} \mathrm{~d} T}{\mathrm{~d} T} \mathrm{~d}}=\frac{q\langle T\rangle}{q_{\text {total }}}
$$

The fractions $\alpha^{L}$ and $\alpha^{L^{\prime}}$ are generally different, but there are two cases where the identity between them can be established:

- the heats of melting for the different solutes are similar and may be considered temperature independent within the temperature range of work, this is often the case for paraffinic waxes;

- as $T$ approaches $T_{c}, \alpha^{L} \rightarrow \quad{ }^{L} \alpha$ thus the identity is verified for temperatures not far removed from the cloud point, $T_{c}$. Often, for hydrocarbon solutions, most of what is considered to be the solute, crystallises within a narrow temperature range below the cloud point. It is then possible to use $\alpha^{L}$, for $\alpha^{L}$.

Unfortunately, it is not possible to directly access the energies defined as $q\langle T\rangle$ and $q_{\text {total }}$ by means of a DSC measurement. Beside the heat used for melting the solute, the energy for heating the solution and heat effects associated to the mixing in the liquid phase are also being measured.

It is thus necessary to isolate the energy used for melting the solute from the others. To calculate the heat fraction defined by Equation (3), data of the enthalpy variation of a solution between an initial temperature $T_{o}$, where the solution is completely solidified, and a temperature $T$ above the cloud point are used. An example of such data is presented in Figure 1. It can be described by:

$$
\begin{aligned}
h(T)= & h^{A}\left(T<T_{m}^{\text {solv }}\right)+h^{B}\left(T=T_{m}^{\text {solv }}\right) \\
& +h^{C}\left(T_{m}^{\text {solv }}<T \leq T_{c}\right)+h^{D}\left(T_{c}<T\right)
\end{aligned}
$$

with:

$$
\begin{gathered}
h^{A}=m_{\text {solv }}^{\text {total }} \int_{T_{o}}^{T} C_{p s o l v}^{S} \mathrm{~d} T+\sum_{i} m_{\text {slti }}^{\text {total }} \int_{T_{O}}^{T} C_{\text {slti }}^{S} \mathrm{~d} T \\
h^{B}=m_{\text {solv }}^{\text {total }} h_{m}^{\text {solv }}\left\langle T_{m}^{\text {solv }}\right\rangle
\end{gathered}
$$

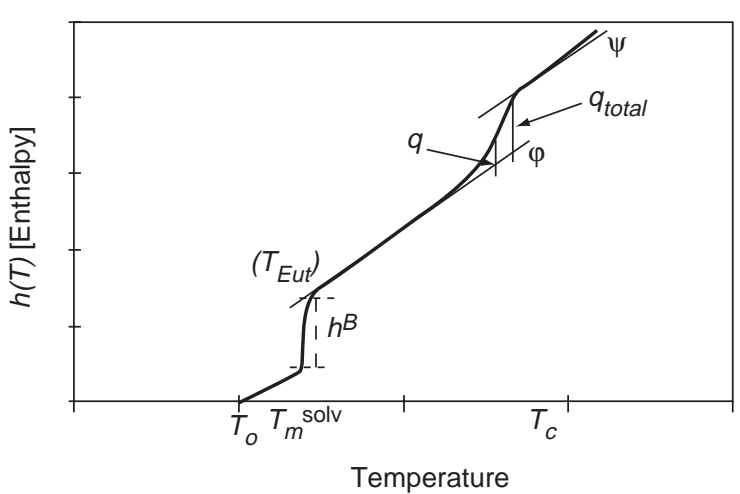

Figure 1

Enthalpy variation of a mixture with the temperature with description of the application of the graphical calculation procedure to estimate the amount of solid formed.

$$
\begin{aligned}
h^{C}= & m_{\text {solv }}^{\text {total }} \int_{T_{m}^{\text {solv }}}^{T} C_{p s o l v}^{L} \mathrm{~d} T+\sum_{i}{ }_{T_{m}^{\text {solv }}}^{T} m_{\text {slti }}^{S} C_{p s l t i}^{S} \mathrm{~d} T \\
& +\sum_{i} \int_{T_{m}^{\text {solv }}}^{T} m_{\text {slti }}^{L} C_{p s l t i}^{L} \mathrm{~d} T+\sum_{i} \int_{T_{m}^{\text {solv }}}^{T} h_{m i}^{\text {slt }} \frac{\mathrm{d} m_{\text {slti }}^{L} \mathrm{~d} T}{\mathrm{~d} T} \\
& +\left(m_{\text {solv }}^{\text {total }}+\sum_{i} m_{\text {slt }}^{L}\right) h_{\text {liq }}^{E} \\
& +\left(\sum_{i} m_{\text {slti }}^{S}\langle T\rangle h_{\text {sld }}^{E}-\sum_{i} m_{\text {slti }}^{\text {total }}\left\langle T_{m}^{\text {solv }}\right\rangle h_{\text {sld }}^{E}\right) \\
& h^{D}=m_{\text {solv }}^{\text {total }} \int_{T_{c}}^{T} C_{p s o l v}^{L} \mathrm{~d} T+\sum_{i} m_{\text {slti }}^{\text {tot }}{ }_{T_{c}}^{{ }^{T}} C_{p s l t i}^{L} \mathrm{~d} T
\end{aligned}
$$

where $P\langle T\rangle$ is the value of the property at temperature $T$. The meaning of the other symbols is the usual or is described in the nomenclature section. The effects due to the excess heat capacities in both phases are neglected. They are too small comparing to the others and normally inferior to the sensibility of the measurements.

To measure $\alpha^{L}$ an auxiliary quantity, $I(T)$, is defined. It represents the energy required to heat the mixture from the solvent melting point to the temperature $T$ and the heat effects associated to the solutes and solvent liquid and solid phases mixing:

$$
\begin{aligned}
I(T)= & h\left\langle T_{m}^{\text {solv }}\right\rangle+m_{\text {solv }}^{\text {total }} \int_{T_{m}^{\text {solv }}}^{T} C_{p s o l v}^{L} \mathrm{~d} T+\sum_{i}{ }_{T_{m}^{\text {solv }}}^{T} m_{\text {slti }}^{S} C_{p s l i}^{S} \mathrm{~d} T \\
& +\sum_{i} \int_{T_{m}^{\text {solv }}}^{T} m_{\text {slti }}^{L} C_{p s l t i}^{L} \mathrm{~d} T+\left(m_{\text {solv }}^{\text {total }}+\sum_{i} m_{\text {slt }}^{L}\right) h_{\text {liq }}^{E} \\
& +\left(\sum_{i} m_{\text {slti }}^{S}\langle T\rangle h_{\text {sld }}^{E}-\sum_{i} m_{\text {slti }}^{\text {total }}\left\langle T_{m}^{\text {solv }}\right\rangle h_{\text {sld }}^{E}\right)
\end{aligned}
$$


Using the energy line $I(T)$, Equation (3) can be represented in terms of the measured quantities by:

$$
\alpha^{L}=\frac{h(T)-I(T)}{h\left(T_{c}\right)-I\left(T_{c}\right)}
$$

To estimate $I(T)$ from the measured data a straight line $\varphi$, tangent to the curve $h(T)$ just above the melting point of the solvent, and another straight line, $\psi$, tangent to the enthalpy curve $h(T)$, just above the temperature of disappearing of the last crystal, $T_{c}$, are drawn. The first is the energy required to heat the liquid solvent and the solid solutes at temperatures above the melting point of the solvent:

$$
\begin{aligned}
\varphi(T)= & h\left\langle T_{m}^{\text {solv }}\right\rangle+\left(m_{\text {solv }}^{\text {total }} C_{p s o l v}^{L}\left\langle T_{m}^{\text {solv }}\right\rangle+\sum_{i} m_{\text {slti }}^{\text {total }} C_{p s l i t}^{S}\left\langle T_{m}^{\text {solv }}\right\rangle\right) \\
& \left(T-T_{m}^{\text {solv }}\right)
\end{aligned}
$$

and the latter the energy used to heat the solution above the cloud point temperature:

$$
\begin{aligned}
\psi(T)= & h\left\langle T_{c}\right\rangle+\left(m_{\text {solv }}^{\text {total }} C_{p s o l v}^{L}\left\langle T_{c}\right\rangle+\sum_{i} m_{\text {slti }}^{\text {total }} C_{p s l t i}^{L}\left\langle T_{c}\right\rangle\right) \\
& \left(T-T_{c}\right)
\end{aligned}
$$

If the heat capacities are considered temperature independent, it is possible to show that:

$$
\begin{aligned}
\frac{\mathrm{d} I}{\mathrm{~d} T}= & \alpha^{L}\left(\frac{\mathrm{d} \psi}{\mathrm{d} T}-\frac{\mathrm{d} \varphi}{\mathrm{d} T}\right)+\frac{\mathrm{d} \varphi}{\mathrm{d} T}+m_{\text {slt }}^{\text {total }} \frac{\mathrm{d} \alpha^{L}}{\mathrm{~d} T}\left(h_{\text {liq }}^{E}-h_{\text {sld }}^{E}\right) \\
& +m_{\text {liq }}^{\text {total }}\langle T\rangle \frac{\mathrm{d} h_{\text {liq }}^{E}}{\mathrm{~d} T}+m_{\text {sld }}^{\text {total }}\langle T\rangle \frac{\mathrm{d} h_{\text {sld }}^{E}}{\mathrm{~d} T}
\end{aligned}
$$

The last three terms disappear if the liquid solution is athermal and there is no solid solution formation. Even when this is not verified, if the liquid and solid phases present the same kind of deviations to the ideality in what concerns the excess enthalpy these terms will compensate each other, strongly decreasing their importance.

Whenever the excess enthalpy effects can be neglected, the estimation of $\alpha^{L}$ is straightforward. Using the experimental slopes of the lines $\varphi$ and $\psi$ the numerical integration of $\mathrm{d} I / \mathrm{d} T$ is performed with a simple iterative procedure providing $\alpha^{L}(T)$.

The assumption, made above, of temperature independent heat capacities affects only the term $(\mathrm{d} \varphi / \mathrm{d} T-\mathrm{d} \psi / \mathrm{d} T)$, but the slopes of the two straight lines are normally similar. Moreover, $\alpha^{L}$ ranges from 0 to 1 further reducing its effect. For the systems studied during the development of this method, this assumption never affected the value of $\mathrm{d} I / \mathrm{d} T$ by more than $2 \%$ and its influence in the estimation of $\alpha^{L}$ is inferior to $0.1 \%$ for $\alpha^{L}>0.01$.
When the difference between the slopes of $\varphi$ and $\psi$ is inferior to 20\%, Equation (13) reduces to $I=\varphi$. Making $I=\varphi$ would in general introduce a small error (typically inferior to $2.5 \%$ in $\alpha^{L}$ for $\alpha^{L}>0.01$ ) but would allow the fraction of melted solute to be calculated from a simple graphical construction using the DSC curve. Being $I=\varphi$, the numerator of Equation (10) is now the distance between the enthalpy curve, $h(T)$, and the straight line $\varphi$, and the denominator the distance between the enthalpy curve and the line $\varphi$ at the temperature of disappearance of the last crystal $T_{c}$. The fraction of solute melted, $\alpha^{L}$, is thus simply given by the ratio of the distance between the enthalpy curve and the line $\varphi$ measured at temperatures $T$ and $T_{c}$ as shown in Figure 1. This gives a simple and fast method to estimate the amount of solid paraffins at a given temperature once a calorimetric measurement as been performed [4].

Examples of the use of the proposed method and its graphical version, for estimation of the amount of solid phase being formed in binary and multicomponent hydrocarbon mixtures, are presented in a later section and the results compared with data measured by alternative techniques.

\section{MEASUREMENT OF BINARY PHASE DIAGRAMS}

The measurement of phase diagrams will use the approach sketched above followed by a conversion of the solid fraction into phase boundaries using the lever rule.

Consider a binary system presenting:

- no solubility in the solid phase, and complete miscibility in the liquid phase with a phase diagram such as the depicted in Figure 2.

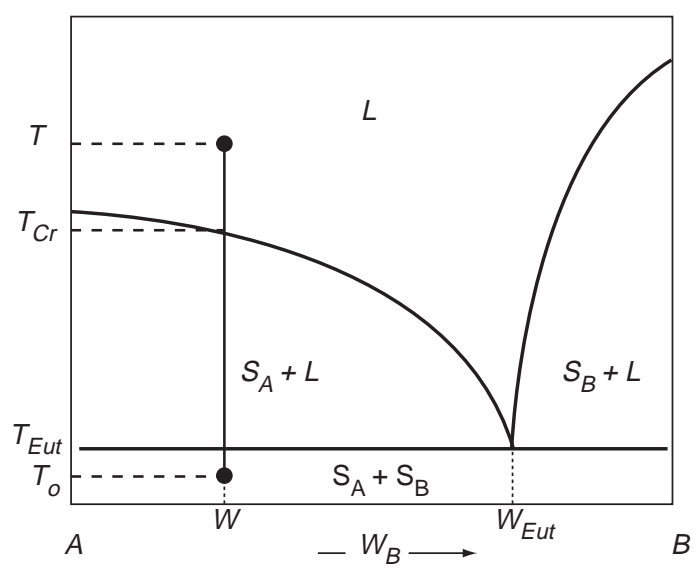

Figure 2

Solid-liquid phase diagram with an eutectic point and complete immiscibility in the solid phase. 
A mixture of composition $W$ in component $B$, at a temperature $T_{o}$ inferior to $T_{E u t}$ is heated until complete fusion. (Unless otherwise stated the compositions always refer to component $B$ ). The enthalpy variation with the temperature during the process is qualitatively identical to what was previously presented in Figure 1.

The first step in the calorimetric analysis would be to check the position of the mixture relatively to the eutectic. The procedure to use for that purpose is outlined below. For the moment, it will be assumed that $0<W<W_{E u t}$. The enthalpy variation is described by Equations (4-8), that for two components simplify into:

$$
\begin{aligned}
h(T)= & h^{a}\left(T<T_{E u t}\right)+h^{b}\left(T=T_{E u t}\right) \\
& +h^{c}\left(T_{E u t}<T \leq T_{c}\right)+h^{d}\left(T_{c}<T\right)
\end{aligned}
$$

with:

$$
\begin{aligned}
& h^{a}=m_{A}^{\text {total }} \int_{T_{O}}^{T} C_{p A}^{S} \mathrm{~d} T+m_{B}^{\text {tot } \int_{T_{O}}}{ }_{T_{p B}}^{T} C_{P}^{S} \mathrm{~d} T \\
& h^{b}=m_{A}^{\text {LEut }} h_{m_{A}}\left\langle T_{\text {Eut }}\right\rangle+m_{B}^{\text {total }} h_{m_{B}}\left\langle T_{\text {Eut }}\right\rangle \\
& +\left(m_{A}^{\text {LEut }}+m_{B}^{\text {total }}\right) h^{E} \\
& h^{c}=m_{B}^{\text {total }} \int_{T_{\text {Eut }}}^{T} C_{p B}^{L} \mathrm{~d} T f_{T_{\text {Eut }}}^{T} m_{A}^{S} C_{p A}^{S} \mathrm{~d} T \\
& +\int_{T_{\text {Eut }}}^{T} m_{A}^{L} C_{p A}^{L} \mathrm{~d} T f_{T_{\text {Eut }}}^{T} h_{m A} \frac{d m_{A}^{L}}{d T} \mathrm{~d} T \\
& +\left(m_{B}^{\text {total }}+m_{A}^{L}\langle T\rangle\right) h^{E}-\left(m_{B}^{\text {total }}+m_{A}^{L E u t}\right) h^{E} \\
& h^{D}=m_{A}^{\text {total }} \int_{T_{c}}^{T} C_{p_{A}}^{L} \mathrm{~d} T+m_{B}^{\text {tot } f l}{ }_{T_{c}}^{T} C_{p_{B}}^{L} \mathrm{~d} T
\end{aligned}
$$

As before the aim is to measure:

$$
\alpha^{L}=\frac{\int_{T_{E u t}}^{T} \frac{\mathrm{d} m_{A}^{L}}{\mathrm{~d} T} \mathrm{~d} T}{\int_{T_{E u t}}^{T_{C}} \frac{\mathrm{d} m_{A}^{L}}{\mathrm{~d} T} \mathrm{~d} T}=\frac{m_{A}^{L}}{m_{A}^{\text {total }}}
$$

that is converted into a phase diagram using the lever rule. The relation between the composition at the phase boundaries, $W^{L}$, and the liquid fraction, $\alpha_{A}{ }^{L}$, is given by:

$$
\begin{aligned}
W^{L} & =\frac{W}{W+(1-W) \alpha_{A}^{L}} \text { for } 0<W<W_{E u t} \\
W^{L} & =\frac{\alpha_{B}^{L} W}{1+W\left(\alpha_{B}^{L}-1\right)} \text { for } W_{E u t}<W<1
\end{aligned}
$$

Fusion of component $A$ takes place in two steps: a portion melts at the eutectic point, $m_{A}{ }^{L E u t}$, and another between $T_{E u t}$ and $T_{c}, m_{A}{ }^{L *}(T)$. It is useful to define two accessory liquid fractions:

$$
\begin{gathered}
\alpha_{A}^{L E u t}=\frac{m_{A}^{L E u t}}{m_{A}^{\text {total }}} \\
\alpha_{A}^{L^{*}}=\frac{m_{A}^{L *}}{m_{A}^{\text {SEut }}}
\end{gathered}
$$

where $m_{A}{ }^{\text {SEut }}=m_{A}{ }^{\text {total }}-m_{A}{ }^{\text {LEut }}$. The relation between these three liquid fractions is:

$$
\alpha_{A}^{L}=\alpha_{A}^{L E u t}+\alpha_{A}^{L *}\left(1-\alpha_{A}^{L E u t}\right)
$$

Considering that $\alpha_{A}^{L^{*}}$ is essentially identical to $\alpha^{L}$, its estimation will be first approached. Using the identity between Equations (2) and (3), $\alpha_{A}{ }^{L^{*}}$ is related to the measured enthalpy variations by:

$$
\alpha_{A}^{L *}=\frac{h(T)-I(T)}{h\left(T_{c}\right)-I\left(T_{c}\right)}
$$

being $I(T)$ the energy required to heat the mixture from the eutectic point to temperature $T$ and the heat effects associated to the mixing of solute and solvent in the liquid phase:

$$
\begin{aligned}
I(T)= & h\left\langle T_{\text {Eut }}\right\rangle+m_{B}^{\text {total }} \int_{T_{\text {Eut }}}^{T} C_{p B}^{L} d T f_{T_{\text {Eut }}}^{T} m_{A}^{S} C_{p A 1}^{S} d T \\
& +\int_{T_{\text {Eut }}}^{T} m_{A}^{L} C_{p A}^{L} d T+\left(m_{B}^{\text {total }}+m_{A}^{L}\langle T\rangle\right) h^{E}
\end{aligned}
$$

To estimate $I(T)$ from the measured data the approach used above will be followed. A straight line $\varphi$, tangent to the enthalpy curve just above the eutectic point, and another straight line, $\psi$, tangent to the curve at the temperature of disappearing of the last crystal, the cloud point temperature, $T_{c}$, are drawn. If the heat capacities are considered to be approximately temperature independent then:

$$
\frac{\mathrm{d} I}{\mathrm{~d} T}=\frac{\mathrm{d} \varphi}{\mathrm{d} T}+\alpha_{A}^{L *}\left(\frac{\mathrm{d} \psi}{\mathrm{d} T}-\frac{\mathrm{d} \varphi}{\mathrm{d} T}\right)+\frac{1}{\alpha_{A}^{L *}} \frac{\mathrm{d} \alpha_{A}^{L^{*}}}{\mathrm{~d} T} h_{E} m_{B}^{\text {total }}
$$

For athermal solutions or solutions with a low heat of mixing, the last term of $\mathrm{d} I / \mathrm{d} T$ can be neglected without a significant effect on $\alpha_{A}{ }^{L^{*}}$. Even for mixtures with higher excess enthalpies this term is usually negligible because $h^{E}$ and $\left(1 / \alpha_{A}{ }^{L^{*}} * \mathrm{~d} \alpha_{A}{ }^{L^{*}} / \mathrm{d} T\right)$ have, for paraffins, opposite variations with the temperature, cancelling each other. If the last term is neglected, knowing that $I\left\langle T_{\text {Eut }}\right\rangle=h\left\langle T_{\text {Eut }}\right\rangle$ the numerical integration of $\mathrm{d} I / \mathrm{d} T$ can be readily performed and, with a simple iterative procedure, the value of $\alpha_{A}{ }^{L^{*}}$ calculated. 
Known $\alpha_{A}{ }^{L^{*}}$, the next step is to calculate $\alpha_{A}{ }^{\text {LEut }}$ and obtain $\alpha_{A}{ }^{L}$ from Equation (19). The definition of a total liquid fraction at the eutectic temperature, $\alpha_{\text {total }}^{\text {LEut }}$,

$$
\alpha_{\text {total }}^{\text {LEut }}=\frac{m_{A}^{L E u t}+m_{B}^{\text {total }}}{m_{A}^{\text {total }}+m_{B}^{\text {total }}}
$$

is helpful because this quantity is related with $\alpha_{A}^{L E u t}$ by:

$$
\alpha_{A}^{L E u t}=\frac{\alpha_{t o t a l}^{L E u t}-W}{1-W}
$$

and from Equations (6a) and (24) it is possible to relate $\alpha_{\text {total }}^{\text {LEut }}$ to measurable quantities:

$$
\alpha_{\text {total }}^{\text {LEut }}=\frac{\frac{h^{b}}{m_{\text {total }}}-W\left(h_{m B}-h_{m A}\right)}{h_{m A}+h^{E}}
$$

where $m_{\text {total }}$ is the mass of the sample being analysed and the heats of fusion are those at the eutectic temperature. Since normally $h_{m A} \gg h^{E}$ the total liquid fraction at the eutectic point can immediately be calculated and from it $\alpha_{A}{ }^{L}$. Moreover, $\alpha_{\text {total }}^{\text {LEut }}$ presents another interest. If the lever rule is applied to the eutectic point, using Equation (23) and some algebraic manipulation, it is possible to relate $\alpha_{\text {total }}^{\text {LEut }}$ to the composition of the eutectic point:

$$
W_{\text {Eut }}=\frac{W}{\alpha_{\text {total }}^{\text {LEut }}}
$$

This means that the eutectic point is completely defined with a single calorimetric measurement. Its temperature is obtained from the "jump" on the enthalpy plot (Fig. 1) and the composition from the total liquid fraction at the eutectic point, $\alpha_{\text {total }}^{\text {LEut }}$, given by Equation (25).

The equations presented above are valid only for $0<W<W_{\text {Eut. }}$ For this reason, before making the analysis of a calorimetric measurement, the eutectic point must be located to know how to perform the analysis correctly. This is done using the Equations (25) and (26), together with the following couple of equations valid for $W_{E u t}<W<1$ :

$$
\alpha_{\text {total }}^{\text {LEut }}=\frac{\frac{h^{b}}{m_{\text {total }}}+(1-W)\left(h_{m B}-h_{m A}\right)}{h_{m B}+h^{E}}
$$

and:

$$
W_{E u t}=1-\frac{(1-W)}{\alpha_{\text {total }}^{\text {LEut }}}
$$

Using these four equations is possible to calculate the composition at the eutectic point since only one of the couples will produce a physically meaningful result. The placement of the mixture relatively to the eutectic point indicates the procedure to be used in the estimation of $\alpha_{A}{ }^{L}$. If $W_{E u t}<W<1$, the method is similar to that described above but the indexes referring to the compounds have to be changed.

This approach should not be restricted to this kind of simple phase diagrams. In principle, it could be applied to more complex phase diagrams presenting intermediate compounds with congruent and incongruent melting points, as long as no solid solutions are formed [5].

\section{DSC MEASUREMENTS}

To show the capacities of the proposed methods to measure the amount of wax deposition and binary phase diagrams, some DSC measurements where performed in several mixtures and the data compared with literature data obtained using other techniques.

The mixtures were prepared from commercially available pure $n$-alkanes (Fluka with a purity of at least 99\%), and ethylbenzene (Aldrich, 99.8\%).

Aluminium pans of $150 \mu \mathrm{l}$ were filled up to $2 / 3$ with the mixtures under study and were analysed in a Mettler TA 3000 differential scanning calorimeter (DSC) after being equilibrated for at least two days at $240 \mathrm{~K}$. At this temperature at least $99 \%$ of the paraffinic material is crystallised. This equilibration reduces the problems associated with nonequilibrium in the solid phase. The DSC has a refrigeration system using liquid nitrogen that allows measurements to be done at temperatures down to $150 \mathrm{~K}$ and provides a very precise temperature control. The sample is cooled from 240 to $150 \mathrm{~K}$ in the calorimeter and, after equilibration, heated from $150 \mathrm{~K}$ up to $320 \mathrm{~K}$ at a rate of $0.5 \mathrm{~K} / \mathrm{min}$. The use of a low heating rate is very important to assure an equilibrium state during the heating. Fusion rather than crystallisation is chosen to avoid undercooling of the solution and problems of solid phase nonequilibrium.

The enthalpy changes were registered every $0.5 \mathrm{~K}$ between the starting and final temperatures and treated according to the proposed procedure.

\section{RESULTS}

From the set of data measured during this work, the results for two hydrocarbon mixtures are presented: a binary with a single solute, docosane, and a mixture of ten $n$-alkanes and an aromatic compound, fluorene. In both cases, the solvent is ethylbenzene. The compositions of the mixtures are presented in Table 1 along with the thermophysical properties of the compounds. The heats of melting are not very different but the possibility of using $\alpha^{L^{\prime}}$ for $\alpha^{L}$ arises mainly from the crystallisation of most of the solute occurring in a narrow temperature range below the cloud point as is manifest from Figures 3 and 4 . The excess 
enthalpies for all these mixtures present positive deviations to the ideality in both the liquid and solid phases. For the binary, the solid fractions measured by calorimetry are compared with those calculated from the phase diagram presented by Ghogomu et al. [6]. For the other mixture, the solid fractions are compared with data determined by crystallisation [3]. There is, in both cases, a very good agreement between the data obtained by the two different procedures giving a good indication about the capacities of the proposed method to measure the solid fractions. Despite of the very large values for the excess enthalpy in the solid phase presented by these systems the assumption that the last terms of Equation (13) are negligible does not seem to introduce a significant error in the measurements.

TABLE 1

Composition (mass \%) of the studied solutions and thermophysical properties of the pure compounds

\begin{tabular}{l|c|c|c|c|c|c}
\hline & Sol. I & Sol. II & $* T_{m}(\mathrm{~K})$ & $* T_{t r}(\mathrm{~K})$ & $\begin{array}{c}* H_{m} \\
(\mathrm{~kJ} / \mathrm{mol})\end{array}$ & $\begin{array}{c}* H_{t r} \\
(\mathrm{~kJ} / \mathrm{mol})\end{array}$ \\
\hline $\begin{array}{l}\text { Ethyl- } \\
\text { benzene }\end{array}$ & 78.56 & 76.75 & 178.2 & & 9.184 & \\
$n-\mathrm{C}_{19}$ & & 3.207 & 305.2 & 295.2 & 45.845 & 13.816 \\
$n-\mathrm{C}_{20}$ & & 2.836 & 310.0 & 300.2 & 46.561 & 15.596 \\
$n-\mathrm{C}_{21}$ & & 2.463 & 313.4 & 305.7 & 47.729 & 15.491 \\
$n-\mathrm{C}_{22}$ & 21.44 & 2.176 & 317.2 & 309.4 & 50.803 & 19.305 \\
$n-\mathrm{C}_{23}$ & & 1.918 & 320.7 & 313.2 & 52.900 & 22.048 \\
$n-\mathrm{C}_{24}$ & & 1.690 & 323.8 & 317.3 & 55.014 & 23.647 \\
$n-\mathrm{C}_{25}$ & & 1.480 & 326.7 & 320.2 & 57.778 & 26.084 \\
$n-\mathrm{C}_{26}$ & & 1.310 & 329.5 & 324.0 & 59.536 & 26.620 \\
$n-\mathrm{C}_{27}$ & & 1.152 & 332.0 & 326.2 & 60.457 & 28.973 \\
$n-\mathrm{C}_{28}$ & & 1.020 & 334.4 & 329.7 & 64.058 & 29.123 \\
Fluorene & & 3.998 & 387.9 & & 19.580 & \\
\hline
\end{tabular}

* data extracted from [7]

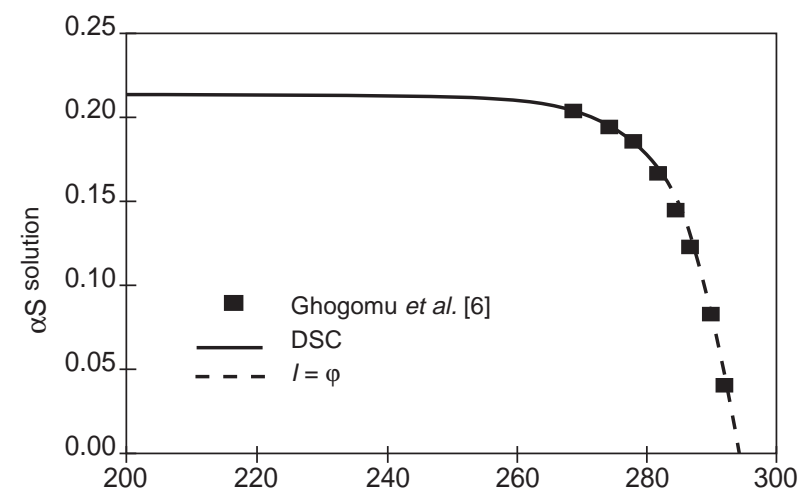

Figure 3

Comparison between the fraction of crystallised solution measured by the proposed method and the data by Ghogomu et al. [6] for solution $\mathrm{I}\left(n-\mathrm{C}_{22} \mathrm{H}_{46}\right.$ /ethylbenzene).

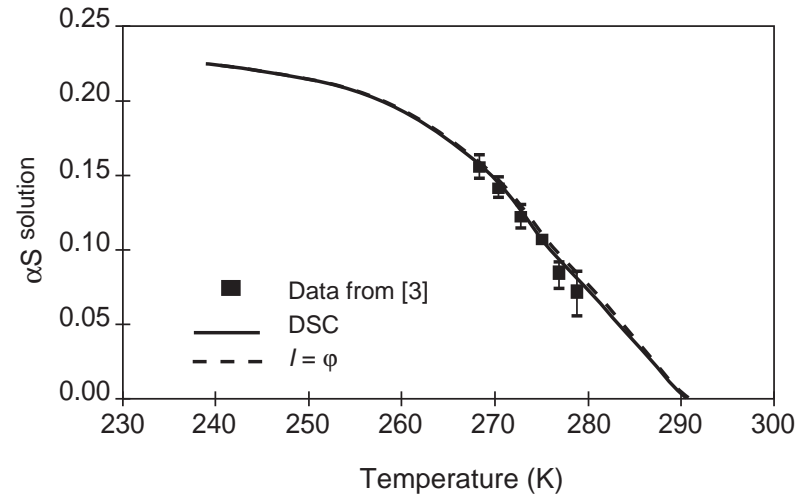

Figure 4

Comparison between the fraction of crystallised solution measured by the proposed method and data from [3] for a mixture of hydrocarbons in ethylbenzene (solution II).

In these figures are also presented the results obtained from the graphical method described before. As shown in Table 2 the slopes of the tangents $\varphi$ and $\psi$ are close enough to make $I=\varphi$. The results presented clearly indicate that the errors introduced in the estimation of the solid fractions by the graphical method are unimportant. This method is of much simpler and faster utilisation, and it can replace in most cases the iterative calculation procedure.

TABLE 2

Slopes of the lines $\varphi$ and $\psi(\mathrm{mJ} / \mathrm{mol} \mathrm{K})$

\begin{tabular}{l|c|c}
\hline & Solution I & Solution II \\
\hline Line $\varphi$ & 92.51 & 90.65 \\
Line $\psi$ & 100.11 & 102.41 \\
\hline
\end{tabular}

The complex solution II was choosen to emulate the paraffins composition of a real fluid in what concerns both the total paraffins content as their distribution with carbon number. Although a real fluid would be much more complex, in particular in what concerns the solvent, the heavy fraction should well illustrate what happens in a real fluid such as a diesel or a fuel. Recent unpublished results of the application of this measurement procedure to diesels indicate that this was a correct approach. The procedure can be extended to real fluids and the mixture used could provide a fair image of what was happening in a diesel.

As for the phase diagram measurement, the analysis of the position of the eutectic point indicates that $W_{E B \text { Eut }}>0.99$. For calculation purposes it was considered that $W_{E B}=1$. The measured phase diagram is shown in Figure 5. Data for the system docosane/ethylbenzene is compared with data by Ghogomu et al. [6]. There is a very good agreement between the two sets of data showing the potential of the proposed technique in solid/liquid phase diagram measurements. 


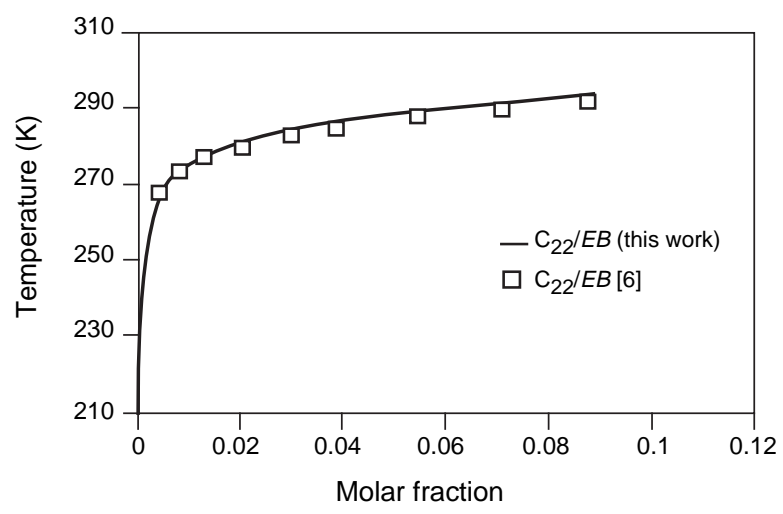

Figure 5

Solid-liquid phase diagrams for docosane/ethylbenzene measured by DSC and data from [6].

\section{CONCLUSIONS}

It was shown how, using DSC measurements, to assess two properties of interest in studies of wax deposition: the amount of solids precipitated and binary SLE phase diagrams of paraffins in a solvent.

Experimental data measured using the proposed methods present a very good agreement with data obtained by other techniques. The new method has the advantage of being much faster and cheaper than conventional methods. With a single DSC measurement the whole range of temperatures of interest may be covered and data concerning these temperatures obtained instead of a lengthsome establishment of equilibrium at each temperature of interest.
A graphical version of the calculation procedure is also presented. The graphical version is very easy to use and is shown to give quick and reliable estimates for paraffin deposition.

While being of a more limited applicability and somewhat lower accuracy than other methods currently used, the proposed procedure to phase diagram measu-rement is much faster and cheaper since a single DSC run is enough to define the phase diagram in the region between two invariant points. It could either be used to directly establish a phase diagram or as a complement to any other phase diagram measurement technique helping to establish the coherence of the measurements, identify and eliminate systematic deviations, or to speed up these measurements by identifying the composition of the invariant points, such as the eutectics, peritectics, or the intermediate compounds compositions [5].

\section{REFERENCES}

1 Wesdorp, L.H. (1990) Liquid-Multiple Solid Phase Equilibrium in Fats. PhD Thesis, TU Delft.

2 Van Winkle, T.L., Affens, W.A., Beal, E.J., Mushrush, G.W., Hazlett, R.N. and DeGuzman, J. (1987) Fuel, 66, 890.

3 Coutinho, J.A.P. and Ruffier-Meray, V., (1997) I\&EC Res., 36, 4977.

4 Coutinho, J.A.P., Calange, S. and Ruffier-Meray, V. (1997) Can. J. Chem. Eng., 75, 1075.

5 Coutinho, J.A.P. and Ruffier-Meray, V. (1998) Fluid Phase Equilibria, in press.

6 Ghogomu, P.M., Dellacherie, J., Balesdent, D. and Chem, J. (1989) Thermodynamics, 21, 925.

7 Broadhurst, M.G. and Res, J. (1962) Nat. Bur. Stand. 66A, 241.

Final manuscript received in August 1999 